\title{
On series of functions with the Baire property
}

\section{Władysław Wilczyński ${ }^{1}$}

Received: 22 January 2018 / Accepted: 16 June 2018 / Published online: 25 July 2018 (c) The Author(s) 2018

\begin{abstract}
Hugo Steinhaus in 1928 proved three theorems concerning convergence almost everywhere and divergence of series of measurable functions. We investigate similarities and differences in the behaviour of sequences of functions with the Baire property for convergence everywhere except a set of the first category.
\end{abstract}

Keywords Series of functions · Measurability $\cdot$ Baire property

Mathematics Subject Classification $40 \mathrm{~A} 30 \cdot 28 \mathrm{~A} 20 \cdot 26 \mathrm{~A} 21$

Recall three classical theorems concerning series of real numbers.

Theorem 1 (Abel and Dini, [2, Chapter IX, Section 39, (173)]) If $\sum_{k=1}^{\infty} a_{k}=+\infty$, $a_{k} \geqslant 0$ for $k \in \mathbb{N}$, then there exists a sequence $\left\{\lambda_{k}\right\}_{k \in \mathbb{N}}$ of positive numbers such that $\lim _{k \rightarrow \infty} \lambda_{k}=0$ and $\sum_{k=1}^{\infty} \lambda_{k} a_{k}=+\infty$.

Theorem 2 (Dini, [2, Chapter IX, Section 39, (175), 4 and Section 41, (178), A]) If $\sum_{k=1}^{\infty} a_{k}<\infty, a_{k} \geqslant 0$ for $k \in \mathbb{N}$, then there exists a sequence $\left\{\lambda_{k}\right\}_{k \in \mathbb{N}}$ of positive numbers such that $\lim _{k \rightarrow \infty} \lambda_{k}=+\infty$ and $\sum_{k=1}^{\infty} \lambda_{k} a_{k}<\infty$.

Theorem 3 (Stieltjes, [2, Chapter IX, Section 41, (178), I]) For each sequence $\left\{\delta_{k}\right\}_{k \in \mathbb{N}}$ of positive numbers such that $\lim _{k \rightarrow \infty} \delta_{k}=0$ there exists a series $\sum_{k=1}^{\infty} a_{k}, a_{k}>0$ for $k \in \mathbb{N}$, such that $\sum_{k=1}^{\infty} a_{k}=+\infty$ and $\sum_{k=1}^{\infty} a_{k} \delta_{k}<\infty$.

These theorems show that there does not exist neither a series which converges slower than any other series nor a series which diverges slower than any other series. This implies among others that no comparison test can be effective with all series.

Theorem 1 remains true also for a sequence $\left\{S_{i}\right\}_{i \in \mathbb{N}}$ of divergent series $\sum_{k=1}^{\infty} a_{i k}$ of positive numbers. Namely, there exists a sequence $\left\{\lambda_{k}\right\}_{k \in \mathbb{N}}$ independent from $i$

Władysław Wilczyński

wwil@uni.lodz.pl

1 Faculty of Mathematics and Computer Science, Łódź University, Banacha 22, 90-238 Łódź, Poland 
such that all series $\sum_{k=1}^{\infty} \lambda_{k} a_{i k}, i \in \mathbb{N}$, are divergent. It was proved in [4,5] with the use of the Banach-Steinhaus theorem. In the same paper Hugo Steinhaus dealt with the following question of Stanisław Ruziewicz: Suppose that a series of positive functions $\sum_{k=1}^{\infty} a_{k}(t), a_{k}:[0,1] \rightarrow \mathbb{R}^{+}$for $k \in \mathbb{N}$, is divergent for each $t \in[0,1]$. Is it possible to find a sequence $\left\{\lambda_{k}\right\}_{k \in \mathbb{N}}$ of positive numbers such that $\lim _{k \rightarrow \infty} \lambda_{k}=0$ and $\sum_{k=1}^{\infty} \lambda_{k} a_{k}(t)=+\infty$ for each $t$ ? The answer is negative, there exists a sequence $\left\{a_{k}(t)\right\}_{k \in \mathbb{N}}$ of measurable functions such that for each sequence $\left\{\lambda_{k}\right\}_{k \in \mathbb{N}}=\Lambda$ there exists a point $x(\Lambda) \in[0,1]$ such that $\sum_{k=1}^{\infty} \lambda_{k} a_{k}(x(\Lambda))<+\infty$.

Let $\boldsymbol{\Lambda}$ be the set of all positive sequences convergent to 0 (card $\boldsymbol{\Lambda}=\mathfrak{C}$ ). Take a set $E \subset[0,1], m(E)=0$, card $E=\mathfrak{C}$ (here $m(\cdot)$ stands for the Lebesgue measure). There exists a function $t: \boldsymbol{\Lambda} \stackrel{1-1}{\longrightarrow} E$ (denotations of Steinhaus [4,5]). For each $\Lambda=$ $\left\{\lambda_{k}\right\}_{k \in \mathbb{N}}$ there exists $\left\{a_{k}^{\Lambda}\right\}_{k \in \mathbb{N}}$ such that $\sum_{k=1}^{\infty} a_{k}^{\Lambda}=+\infty, a_{k}^{\Lambda}>0$, and $\sum_{k=1}^{\infty} \lambda_{k} a_{k}^{\Lambda}<$ $\infty$ (again Stieltjes). Put $a_{k}(t)=a_{k}^{\Lambda}$ for $t=t(\Lambda)$ and $a_{k}(t)=1 / k$ for $t \notin E, k \in \mathbb{N}$. All functions $a_{k}$ are measurable (=1/k a.e.) and

$$
\begin{array}{ll}
\sum_{k=1}^{\infty} a_{k}(t)=+\infty & \text { for each } t \in[0,1], \\
\sum_{k=1}^{\infty} \lambda_{k} a_{k}(t)<\infty & \text { for } t=t(\Lambda), \quad \Lambda=\left\{\lambda_{k}\right\}_{k \in \mathbb{N}} .
\end{array}
$$

If one requires only divergence or convergence almost everywhere, the corresponding versions of Theorems 1, 2, 3 remain true. Three theorems below are also due to Steinhaus $[4,5]$.

Theorem 4 If $\left\{a_{k}(t)\right\}_{k \in \mathbb{N}}$ is a sequence of measurable functions, $a_{k}:[0,1] \rightarrow \mathbb{R}^{+}$ for $k \in \mathbb{N}$ and $\sum_{k=1}^{\infty} a_{k}(t)=+\infty$ almost everywhere on $[0,1]$, then there exists a sequence $\left\{\lambda_{k}\right\}_{k \in \mathbb{N}}$ of positive numbers such that $\lim _{k \rightarrow \infty} \lambda_{k}=0$ and $\sum_{k=1}^{\infty} \lambda_{k} a_{k}(t)=$ $+\infty$ for almost every $t \in[0,1]$.

Theorem 5 If $\left\{a_{k}(t)\right\}_{k \in \mathbb{N}}$ is a sequence of measurable functions, $a_{k}:[0,1] \rightarrow \mathbb{R}^{+}$for $k \in \mathbb{N}$ and $\sum_{k=1}^{\infty} a_{k}(t)<+\infty$ for almost every $t \in[0,1]$, then there exists a sequence $\left\{\lambda_{k}\right\}_{k \in \mathbb{N}}$ of positive numbers such that $\lim _{k \rightarrow \infty} \lambda_{k}=+\infty$ and $\sum_{k=1}^{\infty} \lambda_{k} a_{k}(t)<+\infty$ for almost every $t \in[0,1]$.

Theorem 6 If $\left\{\lambda_{k}(t)\right\}_{k \in \mathbb{N}}$ is a sequence of measurable functions, $\lambda_{k}:[0,1] \rightarrow \mathbb{R}$ for $k \in \mathbb{N}$ and $\lim _{k \rightarrow \infty} \lambda(t)=0$ almost everywhere on $[0,1]$, then there exists a sequence of positive numbers $\left\{a_{k}\right\}_{k \in \mathbb{N}}$ such that $\sum_{k=1}^{\infty} a_{k}=+\infty$ and $\sum_{k=1}^{\infty} a_{k}\left|\lambda_{k}(t)\right|<+\infty$ almost everywhere.

Below we shall prove that Theorem 4 has a satisfactory analogue for the Baire property while Theorems 5 and 6 do not.

Theorem 7 If $\left\{a_{k}(t)\right\}_{k \in \mathbb{N}}$ is a sequence of functions with the Baire property, $a_{k}:[0,1] \rightarrow \mathbb{R}^{+}$for $k \in \mathbb{N}$ and $\sum_{k=1}^{\infty} a_{k}(t)=+\infty$ everywhere except a set of the first category on $[0,1]$, then there exists a sequence $\left\{\lambda_{k}\right\}_{k \in \mathbb{N}}$ of positive numbers such that $\lim _{k \rightarrow \infty} \lambda_{k}=0$ and $\sum_{k=1}^{\infty} \lambda_{k} a_{k}(t)=+\infty$ everywhere except a set of the first category. 
Proof We shall need a simple lemma:

Lemma If $\left\{f_{n}\right\}_{n \in \mathbb{N}}$ is an increasing sequence of functions with the Baire property, $f_{n}:[0,1] \rightarrow \mathbb{R}^{+}$for $n \in \mathbb{N}$ and $\lim _{n \rightarrow \infty} f_{n}(t)=+\infty$ everywhere except a set of the first category, then for each $p>0$ and for each interval $[a, b] \subset[0,1]$ there exists $n_{0} \in \mathbb{N}$ such that $E_{n}=\left\{t \in[a, b]: f_{n}(t) \geqslant p\right\}$ for $n \geqslant n_{0}$ is of the second category (so $E_{n}$ is residual in some subinterval $[c, d] \subset[a, b]$ as a set having the Baire property).

Proof of Lemma Let $E_{n}=\left\{t \in[a, b]: f_{n}(t) \geqslant p\right\}$ for $n \in \mathbb{N}$. Since $\bigcup_{n=1}^{\infty} E_{n}$ is residual in $[a, b]$, there exists $n_{0} \in \mathbb{N}$ such that $E_{n_{0}}$ is of the second category, each $E_{n}$ for $n \geqslant n_{0}$ is also of the second category because the sequence $\left\{E_{n}\right\}_{n \in \mathbb{N}}$ is ascending.

Now, the existence of an increasing sequence of natural numbers $\left\{n_{i}\right\}_{i \in \mathbb{N}}$ will follow from the lemma. Let $n_{1}$ be a natural number for which the set $A_{1}=\{t \in[0,1]$ : $\left.\sum_{k=1}^{n_{1}} a_{k}(t) \geqslant 1\right\}$ is of the second category. Let $n_{2}>n_{1}$ be a natural number for which both sets $A_{2}^{1}=\left\{t \in[0,1 / 2]: \sum_{k=n_{1}+1}^{n_{2}} a_{k}(t) \geqslant 2\right\}, A_{2}^{2}=\{t \in[1 / 2,1]$ : $\left.\sum_{k=n_{1}+1}^{n_{2}} a_{k}(t) \geqslant 2\right\}$ are of the second category. Suppose that we have chosen $n_{1}<$ $n_{2}<\cdots<n_{i}$. Let $n_{i+1}>n_{i}$ be a natural number for which all sets

$$
A_{i+1}^{j}=\left\{t \in\left[\frac{j-1}{2^{i}}, \frac{j}{2^{i}}\right]: \sum_{k=n_{i}+1}^{n_{i+1}} a_{k}(t) \geqslant i+1\right\}, \quad j \in\left\{1,2, \ldots, 2^{i}\right\}
$$

are of the second category.

Now put (similarly as in [4,5]): $\lambda_{k}=1$ for $1 \leqslant k \leqslant n_{1}$ and $\lambda_{k}=1 /(k+1)$ for $n_{i}+1 \leqslant k \leqslant n_{i+1}$. We obviously have $\lim _{k \rightarrow \infty} \lambda_{k}=0$. At the same time $\sum_{k=n_{i}+1}^{n_{i+1}} \lambda_{k} a_{k}(t) \geqslant 1$ for $t \in \bigcup_{j=1}^{2^{i}} A_{i+1}^{j}$. Hence $\sum_{k=1}^{\infty} \lambda_{k} a_{k}(t)=+\infty$ for $t \in$ $\lim \sup _{i \rightarrow \infty}\left(\bigcup_{j=1}^{2^{i}} A_{i+1}^{j}\right)=A$. From the construction it follows that $A$ is residual in $[0,1]$.

To show that analogues of Theorems 5 and 6 do not hold for the Baire property we shall construct a sequence $\left\{f_{n}\right\}_{n \in \mathbb{N}}$ of functions with the Baire property which converges pointwise to zero and does not converge uniformly on any set of the second category with the Baire property. One can find an example of such sequence of functions in [3, Chapter 8], consisting of continuous functions, but in our construction the sequence is non-increasing, which enables us to build a series of functions with non-negative terms.

Let $I_{i}^{n}=\left((i-1) / 2^{n}, i / 2^{n}\right)$ for $n \in \mathbb{N}$ and $i \in\left\{1,2, \ldots, 2^{n}\right\}, A_{n}=\bigcup_{i=1}^{2^{n-1}} I_{2 i-1}^{n}$, $B_{n}=\bigcup_{i=1}^{2^{n-1}} I_{2 i}^{n}$ for $n \in \mathbb{N}$. Put

$$
f_{1}(x)= \begin{cases}1 & \text { for } x \in A_{1} \\ \frac{1}{2} & \text { for } x \in B_{1} \\ 0 & \text { for the remaining } x \in[0,1]\end{cases}
$$


If $f_{1}, f_{2}, \ldots, f_{n-1}$ are already defined, put

$$
f_{n}(x)= \begin{cases}f_{n-1}(x) & \text { for } x \in A_{n}, \\ \frac{1}{n+1} & \text { for } x \in B_{n}, \\ 0 & \text { for the remaining } x \in[0,1] .\end{cases}
$$

Obviously $f_{n+1}(x) \leqslant f_{n}(x)$ for each $n \in \mathbb{N}$ and $x \in[0,1]$. We shall show that $\lim _{n \rightarrow \infty} f_{n}(x)=0$ for each $x \in[0,1]$. Clearly, if the binary expansion of $x$ contains a finite number of 1's (or equivalently, a finite number of 0 's), then $f_{n}(x)=0$ for sufficiently big $n \in \mathbb{N}$. Suppose now that the binary expansion of $x$ contains infinitely many 0 's as well as infinitely many 1 's. If $x=\left(0, a_{1}, a_{2}, \ldots, a_{k}, \ldots\right)_{2}$ and $a_{k}=1$, then from the definition of $f_{k}$ it follows that $f_{k}(x)=1 /(k+1)$; if $a_{k}=0$, then $f_{k}(x)=1 /(j+1)$, where $j=\max \left\{i: a_{i}=1\right.$ and $\left.i<k\right\}$ and $j \rightarrow \infty$ as $k \rightarrow \infty$. Hence $\lim _{n \rightarrow \infty} f_{n}(x)=0$ for all $x \in[0,1]$.

Suppose now that $E \subset[0,1]$ is a set of the second category with the Baire property. Then there exists an interval $(a, b) \subset[0,1]$ and a set $P$ of the first category such that $(a, b) \backslash P \subset E$. We shall show that $\left\{f_{n}\right\}_{n \in \mathbb{N}}$ is not uniformly convergent on $(a, b) \backslash P$. Indeed, there exists $n_{0} \in \mathbb{N}$ such that at least one component of $B_{n_{0}}$ is included in $[a, b]$, so there exists a point $\left.x_{n_{0}} \in B_{n_{0}} \cap((a, b) \backslash P)\right)$ and $f_{n_{0}}\left(x_{n_{0}}\right)=1 /\left(n_{0}+1\right)$. From the construction of $\left\{f_{n}\right\}_{n \in \mathbb{N}}$ it follows that for each $n>n_{0}$ at least one component of $A_{n}$ is included in $(a, b)$, so for each $n>n_{0}$ there exists a point $x_{n} \in A_{n} \cap((a, b) \backslash P)$ such that $f_{n}\left(x_{n}\right)=1 /\left(n_{0}+1\right)$. Hence $\left\{f_{n}\right\}_{n \in \mathbb{N}}$ is not uniformly convergent on $(a, b) \backslash P$ and obviously on $E$.

Theorem 8 There exists a sequence $\left\{a_{k}(t)\right\}_{k \in \mathbb{N}}$ of functions with the Baire property, $a_{k}:[0,1] \rightarrow \mathbb{R}^{+}$for $k \in \mathbb{N}$ and $\sum_{k=1}^{\infty} a_{k}(t)<\infty$ for each $t \in[0,1]$, such that for every sequence $\left\{\lambda_{k}\right\}_{k \in \mathbb{N}}$ of positive numbers tending to infinity the series $\sum_{k=1}^{\infty} \lambda_{k} a_{k}(t)$ is divergent to infinity on a set residual in $[0,1]$.

Proof Put $a_{1}(t)=1-f_{1}(t)$ and $a_{k}(t)=f_{k-1}(t)-f_{k}(t)$ for $k \geqslant 2$, where $\left\{f_{n}\right\}_{n \in \mathbb{N}}$ is the sequences (1)-(2). Then $\sum_{i=1}^{k} a_{i}(t)=1-f_{k}(t)$ for $k \in \mathbb{N}$, so $\sum_{k=1}^{\infty} a_{k}(t)=1$ everywhere except a denumerable set. Observe that $a_{k}(t) \geqslant 1 / k-1 /(k+1)=$ $1 /(k(k+1))$ for $t \in B_{k}$, since $f_{k-1}(t) \geqslant 1 / k$ and $f_{k}(t)=1 /(k+1)$ on $B_{k}$. From the construction of $\left\{f_{n}\right\}_{n \in \mathbb{N}}$ it follows immediately that $a_{k+p}(t) \geqslant 1 /(k(k+1))$ on $A_{k+p} \cap B_{k}$ for each $p \in \mathbb{N}$.

Let $\left\{p_{k}\right\}_{k \in \mathbb{N}}$ be an increasing sequence of natural numbers with $\lambda_{k+p_{k}}>k(k+1)$ for each $k \in \mathbb{N}$. Then we have $\lambda_{k+p_{k}} \cdot a_{k+p_{k}}(t)>1$ for $t \in A_{k+p_{k}} \cap B_{k}$. Hence $\sum_{k=1}^{\infty} \lambda_{k} a_{k}(t) \geqslant \sum_{k=1}^{\infty} \lambda_{k+p_{k}} a_{k+p_{k}}(t)=+\infty$ for $t \in \lim \sup _{k \rightarrow \infty} A_{k+p_{k}} \cap B_{k}$ and the last set is residual in $[0,1]$.

Theorem 9 There exists a sequence $\left\{f_{k}(t)\right\}_{k \in \mathbb{N}}$ of functions with the Baire property, $f_{k}:[0,1] \rightarrow \mathbb{R}^{+}$for $k \in \mathbb{N}$ and $\lim _{k \rightarrow \infty} f_{k}(t)=0$ for $t \in[0,1]$, such that for each sequence $\left\{a_{k}\right\}_{k \in \mathbb{N}}$ of positive numbers with $\sum_{k=1}^{\infty} a_{k}=+\infty$ the series $\sum_{k=1}^{\infty} a_{k} f_{k}(t)$ is divergent to infinity on a set residual in $[0,1]$.

Proof Let $\left\{f_{k}(t)\right\}_{k \in \mathbb{N}}$ be the sequence of functions (1)-(2). Suppose that $\sum_{k=1}^{\infty} a_{k}=$ $+\infty$. There exists $n_{1} \in \mathbb{N}$ such that $\sum_{k=1}^{n_{1}} a_{k}>1$. Observe that $f_{1}(t)=f_{2}(t)=\cdots=$ 
$f_{n_{1}}(t)=1$ for $t \in\left(0,1 / 2^{n_{1}}\right)$, so $\sum_{k=1}^{n_{1}} a_{k} f_{k}>1$ on this interval. There exists $n_{2}>n_{1}$ such that $\sum_{k=n_{1}+1}^{n_{2}} a_{k}>2$. Again observe that $f_{n_{1}+1}(t), f_{n_{1}+2}(t), \ldots, f_{n_{2}}(t) \geqslant 1 / 2$ for $t \in\left(0,1 / 2^{n_{2}}\right) \cup\left(1 / 2,1 / 2+1 / 2^{n_{2}}\right)$ (actually the value of all functions above is equal to 1 in the first interval and to $1 / 2$ in the second one), so $\sum_{k=n_{1}+1}^{n_{2}} a_{k} f_{k}(t)>1$ on this set. Suppose that we have found $n_{1}<n_{2}<\cdots<n_{i}$. Let $n_{i+1}$ be a number such that $\sum_{k=n_{i}+1}^{n_{i+1}} a_{k}>i+1$. Similarly, we observe that $f_{n_{i}+1}(t), f_{n_{i}+2}(t), \ldots, f_{n_{i+1}}(t) \geqslant$ $1 /(i+1)$ on an open set $E_{i}$ such that the intersection of $E_{i}$ with each component of $A_{i+1}$ is nonempty (namely it is equal to the interval $\left(2(j-1) / 2^{i+1}, 2 j-1 / 2^{i+1}\right) \cap$ $\left.\left(2(j-1) / 2^{i+1}, 2(j-1) / 2^{i+1}+1 / 2^{n_{i+1}}\right)\right)$. Hence $\sum_{k=n_{i}+1}^{n_{i+1}} a_{k} f_{k}(t)>1$ for $t \in E_{i}$. Finally $\sum_{k=1}^{\infty} a_{k} f_{k}(t)=+\infty$ for $t \in \limsup _{i \rightarrow \infty} E_{i}$ and the last set is residual in $[0,1]$.

We show another similarity and difference between measure and category. Bartle in [1] proved the following theorem:

Theorem 10 If a sequence $\left\{f_{n}\right\}_{n \in \mathbb{N}}$ of measurable functions on $[0,1]$ converges almost uniformly to $f$, then it satisfies the vanishing restriction with respect to $f$. If $\left\{f_{n}\right\}_{n \in \mathbb{N}}$ converges in measure to $f$ and $\left\{f_{n}\right\}_{n \in \mathbb{N}}$ satisfies the vanishing restriction with respect to $f$, then $\left\{f_{n}\right\}_{n \in \mathbb{N}}$ converges almost uniformly to $f$.

Here the convergence almost uniformly means that there exists a sequence $\left\{B_{i}\right\}_{i \in \mathbb{N}}$ of measurable sets such that $m\left(\bigcup_{i=1}^{\infty} B_{i}\right)=1\left([0,1] \backslash \bigcup_{i=1}^{\infty} B_{i}\right.$ is a nullset) and $f_{n \mid B_{i}} \rightrightarrows f_{\mid B_{i}}$ for each $i \in \mathbb{N}$ as $n \rightarrow \infty$ (cf. Egorov's theorem).

The sequence $\left\{f_{n}\right\}_{n \in \mathbb{N}}$ of measurable functions satisfies the vanishing restriction with respect to $f$ if for all $\alpha>0$ we have

$$
\lim _{n \rightarrow \infty} m\left(E_{n}^{f}(\alpha)\right)=0, \text { where } E_{n}^{f}(\alpha)=\bigcup_{j=n}^{\infty}\left\{x \in[0,1]:\left|f_{j}(x)-f(x)\right|>\alpha\right\}
$$

Since the sequence $\left\{E_{n}^{f}(\alpha)\right\}_{n \in \mathbb{N}}$ is descending, the last condition means $\bigcap_{n=1}^{\infty} E_{n}^{f}(\alpha)$ is a nullset.

We shall say that a sequence $\left\{f_{n}\right\}_{n \in \mathbb{N}}$ of functions with the Baire property converges to $f B$-almost uniformly if there exists a sequence $\left\{B_{i}\right\}_{i \in \mathbb{N}}$ of sets with the Baire property such that $[0,1] \backslash \bigcup_{i=1}^{\infty} B_{i}$ is of the first category and $f_{n \mid B_{i}} \rightrightarrows f_{\mid B_{i}}$ for each $i \in \mathbb{N}$ as $n \rightarrow \infty$. We can (and shall) suppose that the sequence $\left\{B_{i}\right\}_{i \in \mathbb{N}}$ is ascending.

We shall say that a sequence $\left\{f_{n}\right\}_{n \in \mathbb{N}}$ satisfies the $B$-vanishing restriction with respect to $f$ if for all $\alpha>0$ the set $\bigcap_{n=1}^{\infty} E_{n}^{f}(\alpha)$ is of the first category.

Theorem 11 If a sequence $\left\{f_{n}\right\}_{n \in \mathbb{N}}$ of functions with the Baire property on $[0,1]$ converges B-almost uniformly to $f$, then it satisfies the B-vanishing condition with respect to $f$. There exists a sequence $\left\{f_{n}\right\}_{n \in \mathbb{N}}$ of functions with the Baire property on $[0,1]$ which converges everywhere to $f \equiv 0$ and satisfies the $B$-vanishing restriction with respect to $f$ but which does not converge $B$-almost uniformly to $f$.

Proof Suppose that $\left\{B_{i}\right\}_{i \in \mathbb{N}}$ is an increasing sequence of sets with the Baire property such that $[0,1] \backslash \bigcup_{i=1}^{\infty} B_{i}$ is of the first category and $f_{n \mid B_{i}} \rightrightarrows f_{\mid B_{i}}$ for each $i \in \mathbb{N}$ as $n \rightarrow \infty$. 
Fix $\alpha>0$. For each $i \in \mathbb{N}$ there exists $n_{\alpha, i} \in \mathbb{N}$ such that for each $x \in B_{i}$ and $n \geqslant n_{\alpha, i}$ we have $\left|f_{n}(x)-f(x)\right| \leqslant \alpha$. So $E_{n_{\alpha, i}}(\alpha) \subset[0,1] \backslash B_{i}$ and $\bigcap_{n=1}^{\infty} E_{n}(\alpha) \subset$ $\bigcap_{i=1}^{\infty}\left([0,1] \backslash B_{i}\right)=[0,1] \backslash \bigcup_{i=1}^{\infty} B_{i}$ which completes the proof of the first part of the theorem.

To prove the second part it is sufficient to observe that the sequence $\left\{f_{n}\right\}_{n \in \mathbb{N}}$ of functions (1)-(2) satisfies the $B$-vanishing restriction. If this sequence were convergent uniformly to the zero-function on a set $E \subset[0,1]$ with the Baire property, then $E$ would be of the first category. Hence it is clear that $\left\{f_{n}\right\}_{n \in \mathbb{N}}$ does not converge $B$-almost uniformly to the zero-function.

Open Access This article is distributed under the terms of the Creative Commons Attribution 4.0 International License (http://creativecommons.org/licenses/by/4.0/), which permits unrestricted use, distribution, and reproduction in any medium, provided you give appropriate credit to the original author(s) and the source, provide a link to the Creative Commons license, and indicate if changes were made.

\section{References}

1. Bartle, R.G.: An extension of Egorov's theorem. Amer. Math. Mon. 87(8), 628-633 (1980)

2. Knopp, K.: Theory and Application of Infinite Series. Blackie and Son, London (1951)

3. Oxtoby, J.C.: Measure and Category, 2nd edn. Graduate Texts in Mathematics, vol. 2. Springer, New York (1980)

4. Steinhaus, H.: Sur une question concernant la convergence de séries des fonctions. Fund. Math. 11, 186-192 (1928)

5. Steinhaus, H.: Selected Papers, pp. 373-379. PWN, Warsaw (1985) 